\title{
A Phase I Dose Escalation Study of Apatinib Combinated with Docetaxel as Second-line Therapy for Advanced Gastric Carcinoma
}

\author{
Y. ZHENG, WEI WANG, YONG FANG, Q. PAN, ZHEN LIU, J. YAO AND H. PAN*
}

Department of Oncology, Sir Run Run Shaw Hospital, School of Medicine, Zhejiang University, Hangzhou 310016, China

Zheng et al: : Study of Apatinib with Docetaxel as Second-line Therapy for Gastric Carcinoma

This study is a phase I clinical trial of $3+3$ dose escalation. Three dose escalations of apatinib were employed in this study: $425 \mathrm{mg}, 500 \mathrm{mg}$ and $675 \mathrm{mg}$. Apatinib combined with fixed dose of docetaxel (60 $\mathrm{mg} / \mathrm{m}^{2}, \mathrm{~d} 1$, thrice in $\left.\mathbf{w}\right) .21 \mathrm{~d}$ as one treatment cycle was repeated. Maximum tolerated dose, safety and efficacy were analyzed. From October 2015 to June 2017, a total of eight patients with advanced gastric or gastro-oesophageal junction adenocarcinoma who had failed in the previous first-line treatment were enrolled. One dose-limiting toxicity was observed in patients who received apatinib with the dose of 425 mg combined with docetaxel, but no dose-limiting toxicity was found in additional three patients. Two patients treated with apatinib at the dose of $500 \mathbf{~ m g}$ combined with docetaxel experienced dose-limiting toxicity, including grade 3 oral mucositis and grade 4 neutropenia respectively. Therefore, Maximum tolerated dose of apatinib was $425 \mathrm{mg}$. No complete response and partial response were observed; five patients showed stable disease and two had progressive disease. The disease control rate was $71.43 \%$. The median progression free survival was 4.4 mo (95\% confidence interval, 1.48-7.03) and the median overall survival was 11.5 mo (95\% confidence interval, 3.06-16.56). All patients developed adverse events and treatment-related adverse events. Seven patients $(77.78 \%)$ showed treatment-related adverse events greater than or equal to grade 3 , mostly including neutropenia and hand-foot syndrome. The combination of apatinib with the dose of $425 \mathrm{mg}$ and docetaxel was well tolerated and support further evaluation for the second-line treatment of advanced gastric cancer.

Key words: Apatinib, docetaxel, gastric cancer, second-line treatment

Gastric cancer including gastro-oesophageal junction cancer, remains one of the most common malignant and fatal diseases worldwide ${ }^{[1]}$. China has a large burden of gastric cancer, accounting for $44 \%$ of new cases and 50 $\%$ of cancer-related deaths worldwide ${ }^{[2]}$. Target therapy and immunotherapy have progressed in recent years and greatly improved survival for patients after secondline therapy ${ }^{[3-6]}$. However, standard second-line regimens remain primarily docetaxel, paclitaxel and irinotecan and the clinical benefit was limited ${ }^{[7,8]}$. Therefore, it is necessary to explore new second-line treatment for advanced gastric cancer to meet current clinical needs. Apatinib, a small-molecule tyrosine kinase inhibitor (TKI) that highly selectively binds to the intracellular domain of vascular endothelial growth factor receptor 2 (VEGFR-2), inhibits the receptor- mediated pathway activation and suppresses of angiogenesis ${ }^{[4,9,10]}$. Apatinib, as a third-line or higher therapy, has shown significant survival benefits and acceptable safety in patients with advanced or metastatic gastric cancer refractory to chemotherapy in phase II and III trials ${ }^{[4,10]}$. Apatinib was approved in China for metastatic gastric cancer or after second-line chemotherapy for adenocarcinoma of the gastroesophageal junction based on these results. Apatinib in combination with chemotherapy showed high response rate and good tolerability in patients with advanced or metastatic squamous non-small cell lung cancer (NSCLC) ${ }^{[11]}$. Furthermore, a prospective clinical study compared the efficacy and safety of apatinib in combination with chemotherapy versus chemotherapy alone as second or higher line therapy for patients

*Address for correspondence

E-mail: panhongming@zju.edu.cn 
advanced or metastatic gastric cancer. Combination therapy significantly improved progression-free survival (PFS) and disease control rate $(\mathrm{DCR})^{[12]}$. Apatinib combined with chemotherapy also demonstrated improved survival benefit in two other studies $^{[13,14]}$. However, phase 1 dose-escalation studies of apatinib combined with chemotherapy are lacking. In addition, combined chemotherapy regimens in these studies are heterogeneous including docetaxel, paclitaxel, irinotecan and fluorouracil. Therefore, we conducted a study to determine the maximum tolerated dose (MTD) and assess the safety and efficacy of apatinib in combination with docetaxel in the secondline treatment of advanced gastric cancer. Enrollment criteria included the following conditions: Aged $\geq 18$ y; signed informed consent; imaging diagnosis confirmed disease progression after first-line treatment (including 5-fluorouracil and platinum combined chemotherapy regimens); patient's life expectancy $\geq 16 \mathrm{w}$; Eastern Cooperative Oncology Group (ECOG) performance status score $0-1$; adequate bone marrow hematopoietic function and liver and kidney function; at least one measurable lesion. Patients were excluded for the following conditions: receiving prior taxane therapy, including paclitaxel and paclitaxel; patients who have received VEGFR inhibitors, such as sorafenib and sunitinib; patients with hypertension who cannot be brought down to the normal range after treatment; abnormal coagulation function with bleeding tendency; patients with brain metastases. This study was a single center, phase I, open-label, $3+3$ design,dose-escalation study. The primary objective was to determine the MTD of apatinib, and safety of apatinib combined with docetaxel in patients with advanced or metastatic gastric or gastro-oesophageal junction adenocarcinoma after failed the first-line chemotherapy. The secondary objectives were to evaluate efficacy of apatinib in combination with docetaxel in second-line therapy of patients with advanced gastric cancer. The study was conducted in accordance with the Declaration of Helsinki, the International Conference on Harmonization Guideline on Good Clinical Practice and applicable local regulatory requirements and laws. The protocol have been approved by the Ethics Committee at Shao Yifu Hospital Affiliated to Zhejiang University School of Medicine. And all participants provided written informed consent. Apatinib was administered at 425 $\mathrm{mg}, 500 \mathrm{mg}, 675 \mathrm{mg}$ and $750 \mathrm{mg}$ orally once daily in combination with docetaxel $60 \mathrm{mg} / \mathrm{m}^{2}$ intravenously on day 1 every three $w$. Three patients were enrolled at dose of $425 \mathrm{mg}$. If none of three patients developed dose-limiting toxicity (DLT), the dose was continuously increased. If one patient developed DLT, other three patients were enrolled at the same dose, and if none of the other three patients developed DLT, dose escalation continued. If more than two patients develop DLT at one dose, or if anyone of the three additional patients developed DLT, dose escalation stopped, and the previous level was defined as the MTD. All patients continued combination therapy until unacceptable toxicity, disease progression, protocol deviation, death or withdrawal of consent. Toxicity were graded according to the National Cancer Institute Common Terminology Criteria for Adverse Events version version 4.0. DLT was assessed in the first treatment cycle and was defined as grade 4 or more hematologic toxicity or grade 3 thrombocytopenia with bleeding or grade 3 or more non-hematologic toxicity excluding alopecia, nausea and vomiting. Tumor assessments were performed every $6 \mathrm{w}$ according to RECIST (version 1.1). Efficacy endpoints include PFS, overall survival (OS), overall response rate (ORR) and DCR. Survival was followed up every 3 mo until disease progression, death, or receiving other anti-cancer treatment or loss of follow-up. Efficacy and safety analyses were performed on all patients who received at least one dose of the study drugs. Quantitative variables were analyzed by mean, standard deviation, median and interquartile range; qualitative variables were descriptive by number and percentage. PFS and OS were analyzed using the Kaplan-Meier method. Statistical analyses were performed using SPSS for Windows (version 21; IBM, Armonk, New York, USA). A total of eight patients with advanced gastric adenocarcinoma were enrolled in this study, with a median age of $53.5 \mathrm{y}$ (range 27-64 y). Table 1 showed the baseline characteristics of eight eligible patients. Half of patients were male and others were female. All patients had gastric primary lesion with an ECOG performance status of 1 and were at clinical stage IV. Four patients experienced surgery while others did not. Four patients were previously treated with SOX, three with FOLFOX, and one with XELOX regimens, respectively (Table 1). The DLTs were observed day 1-21 at each dose level during the first clycle. Three patients were included at the first dose $(425 \mathrm{mg})$, and one patient experienced DLT at this dose of apatinib due to grade 3 bilirubin elevation. Subsequently, additional three patients were treated at this dose, no DLT was occurred. Three patients were then enrolled at dose of $500 \mathrm{mg}$. Two patients developed grade 3 oral mucositis and grade 4 neutropenia. Therefore, the MTD 
and recommended dose was determined to be $425 \mathrm{mg}$ / day apatinib once daily combined with docetaxel. The median treatment duration was $73.5 \mathrm{~d}$ with interquartile range of 42-115.5 d. All patients had adverse events. More than $1 / 3$ of the patients had neutropenia, leucopenia, upper gastrointestinal hemorrhage, anemia, oral mucositis, alopecia, hypertension, proteinuria, asthenia and hypoalbuminemia. Seven of eight (87.5 $\%$ ) patients showed adverse events $\geq$ grade 3 . The most common more than grade 3 adverse events were neutropenia (25\%), hand-foot syndrome (HFS) (25\%) (Table 2). All patients experienced treatment-related AEs (TRAEs), of which six (75\%) patients had more than grade 3 TRAEs, mostly including neutropenia (25 $\%)$, HFS (25\%) (Table 3). There were no treatmentrelated deaths in this study. Seven of eight patients had efficacy evaluation. None of patients showed complete response (CR) and partial response (PR) with ORR of 0 $\%$. Five patients had stable disease (SD), two patients had disease progression (PD) and DCR was $71.43 \%$ (Table 4). The median PFS was 4.4 mo (95\% confidence interval (CI), 1.48-7.03) and the mdian OS was $11.5 \mathrm{mo}$ (95\% CI, 3.06-16.56) (fig. 1). This is the first phase I study that evaluated the safety and tolerability of apatinib combined with docetaxel in advanced gastric cancer after failing the first-line treatment. Docetaxel has been applied as both the first-line and second-line treatment for advanced gastric cancer ${ }^{[15,16]}$. Additionally, apatinib has been approved for patients with advanced gastric cancer as third-line or more-line treatment ${ }^{[4,10]}$. However, tolerability and efficacy of apatinib in combination with docetaxel and first-rank dose for the patients with advanced gastric cancer remains to be established. Accordingly, this study centred around detecting the MTD of apatinib plus docetaxel in advanced gastric cancer. In this study, the MTD was determined as $425 \mathrm{mg} /$ day apatinib plus $60 \mathrm{mg} / \mathrm{m}^{2}$ docetaxel and the recommended dose for further phase II study. Though we observd a lower apatinib dose of $425 \mathrm{mg}$ for advanced gastric cancer patients than the recommended dose of $850 \mathrm{mg}$ in the phase III trial ${ }^{[4]}$, the patients showed a limit survival benefit with acceptable toxicity. Furthermore, apatinib with a low dose of $425 \mathrm{mg}$ definitely gave rise to less adverse events than those in the recommeded dose of $850 \mathrm{mg}^{[4]}$. For now, the MTD of apatinib have been validated in this combination regimen. Whereas, the most suitable dose of apatinib plus docetaxel for advanced gastric cancer patients should be further identified. Safety analysis found that the most common adverse events at high grade from apatinib combined with docetaxel were neutropenia, hypertension, anemia, oral mucositis, Special Issue 4, 2021 upper gastrointestinal bleeding, which were consistent with the data of phase III trial ${ }^{[4]}$ and there were novel adverse events different from prior reports. Therefore, the toxicity of apatinib was governable and sufferable and could be handled by the interruption timely. In reality, apatinib or docetaxel alone had been reported the known toxicites such as hypertension and neutropenia, respectively. Therefore, apatinib combined with docetaxel could not result in the haematologic toxicity increase compared with either drug alone. Altogether, this phase I study demonstrated that apatinib combined with docetaxel showed acceptable toxicity in patients with advanced gastric cancer. However, a phase II is required to further delinate the real toxicity of the combination therapy with apatinib and docetaxel. The efficacy was not the primary end point of this study, but our data showed that apatinib combined docetaxel improved the median PFS to 4.4 mo and the median OS to $11.5 \mathrm{mo}$ as a second-line therapy, which was different from the previous data on chemotheapy and antiangionenic target therapy. Paclitaxel and irinotecan achieved a median PFS of 2.2-3.6 mo and a median OS of 5.8-9.5 mo as second-line therapy in patients with advanced gastric cancer ${ }^{[8,17]}$. Ramucirumab monotherapy showed a median OS of 5.2 mo and combination with paclitaxel achieved a mdian OS of 9.6 mo in patients with advanced gastric cancer ${ }^{[3,18]}$. Apatinib combined docetaxel demonstrated strong antitumor activity preliminarily. Although the combination of apatinib and docetaxel is working at the recommended phase I dose established in this study, it is unclear the optimal dose and real efficacy according to the phase I trial. A more large scale study with multiple centers would be more convincing. In conclusion, the outcome of this study demonstrated that the MTD and recommended dose of apatinib for phase II study was defined as $425 \mathrm{mg}$. The patients administrated apatinib at the dose of $425 \mathrm{mg}$ combined with docetaxel at 60 $\mathrm{mg} / \mathrm{m}^{2}$ showed tolerated toxicities and similar effectiveness to previous standard second-line therapy. The combination treatment with apatinib and docetaxel may prove to be an effective therapy for patients with advanced gastric cancer.
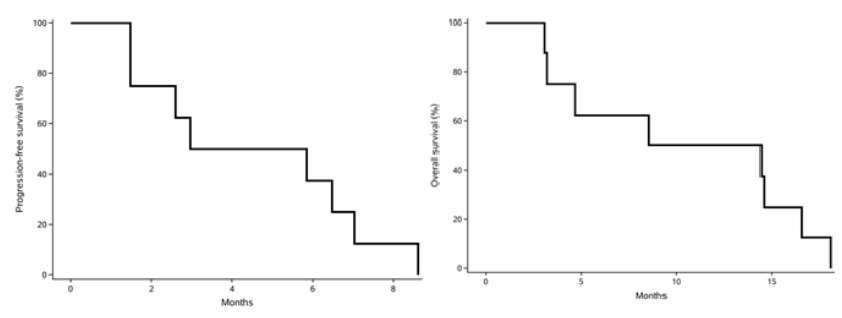

Fig. 1: (A) Kaplan-Meier analysis of progression-free survival and $(B)$ overall survival 
TABLE 1: THE BASELINE OF PATIENTS

\begin{tabular}{|c|c|}
\hline Characteristics & Value \\
\hline \multicolumn{2}{|l|}{ Age $(y)$} \\
\hline Midian, (range) & $53.5(27-64)$ \\
\hline \multicolumn{2}{|l|}{ Sex } \\
\hline Female, n (\%) & $4(50)$ \\
\hline Male, $\mathrm{n}(\%)$ & $4(50)$ \\
\hline \multicolumn{2}{|l|}{$\begin{array}{l}\text { ECOG performance status, } n \\
(\%)\end{array}$} \\
\hline I & $8(100)$ \\
\hline \multicolumn{2}{|l|}{ Stage, n (\%) } \\
\hline IV & $8(100)$ \\
\hline \multicolumn{2}{|l|}{ Histologic grade, n (\%) } \\
\hline Moderately differentiated & $2(25)$ \\
\hline Poorly differentiated & $3(37.5)$ \\
\hline Unknown & $3(37.5)$ \\
\hline \multicolumn{2}{|l|}{ Primary lesion, $\mathrm{n}(\%)$} \\
\hline Gastric & $8(100)$ \\
\hline \multicolumn{2}{|l|}{$\begin{array}{l}\text { Previous surgery for gastric } \\
\text { cancer, } \mathrm{n}(\%)\end{array}$} \\
\hline Yes & $4(50)$ \\
\hline No & $4(50)$ \\
\hline \multicolumn{2}{|l|}{ Previous chemotherapy, n (\%) } \\
\hline sox & $4(25)$ \\
\hline FOLFOX & $3(37.5)$ \\
\hline XELOX & $1(12.5)$ \\
\hline
\end{tabular}

TABLE 2: ADVERSE EVENTS (AES)

\begin{tabular}{|c|c|c|c|c|c|}
\hline \multirow{2}{*}{$\begin{array}{l}\text { FOLFOX } \\
\text { XELOX }\end{array}$} & \multicolumn{2}{|r|}{$3(37.5)$} & & \multirow[b]{2}{*}{ Number $(n=7)$} & \multirow[b]{2}{*}{$\begin{array}{c}\text { Percentage of } \\
\text { patients (\%) }\end{array}$} \\
\hline & & 1 (12.5) & & & \\
\hline \multicolumn{3}{|c|}{ TABLE 2: ADVERSE EVENTS (AEs) } & CR & 0 & 0 \\
\hline AEs & All grades, $\mathrm{n}(\%)$ & Grade $\geq 3, \mathrm{n}(\%)$ & PR & 0 & 0 \\
\hline Neutropenia & $6(75)$ & $2(25)$ & SD & 5 & 71.43 \\
\hline Leukopenia & $6(75)$ & $0(0)$ & PD & 2 & 28.57 \\
\hline \multirow{2}{*}{$\begin{array}{l}\text { Upper } \\
\text { gastrointestinal } \\
\text { hemorrhage }\end{array}$} & \multirow[b]{2}{*}{$5(62.5)$} & \multirow[b]{2}{*}{$1(12.5)$} & ORR & 0 & 0 \\
\hline & & & DCR & 5 & 71.43 \\
\hline
\end{tabular}

hemorrhage

$\begin{array}{lcc}\text { Anemia } & 5(62.5) & 1(12.5) \\ \text { Oral mucositis } & 4(50) & 1(12.5) \\ \text { Alopecia } & 4(50) & 0 \\ \text { Hypertension } & 3(37.5) & 1(12.5) \\ \text { Proteinuria } & 3(37.5) & 0 \\ \text { Asthenia } & 3(37.5) & 0 \\ \text { Hypoalbuminemia } & 3(37.5) & 0 \\ \text { Abdominal pain } & 2(25) & 0 \\ \begin{array}{l}\text { Dizziness } \\ \text { abdominal }\end{array} & 2(25) & 0 \\ \text { distention } & 2(25) & 0 \\ \text { Diabetes } & 2(25) & 0 \\ \begin{array}{l}\text { Hand-foot } \\ \text { syndrome }\end{array} & 2(25) & 2(25)\end{array}$

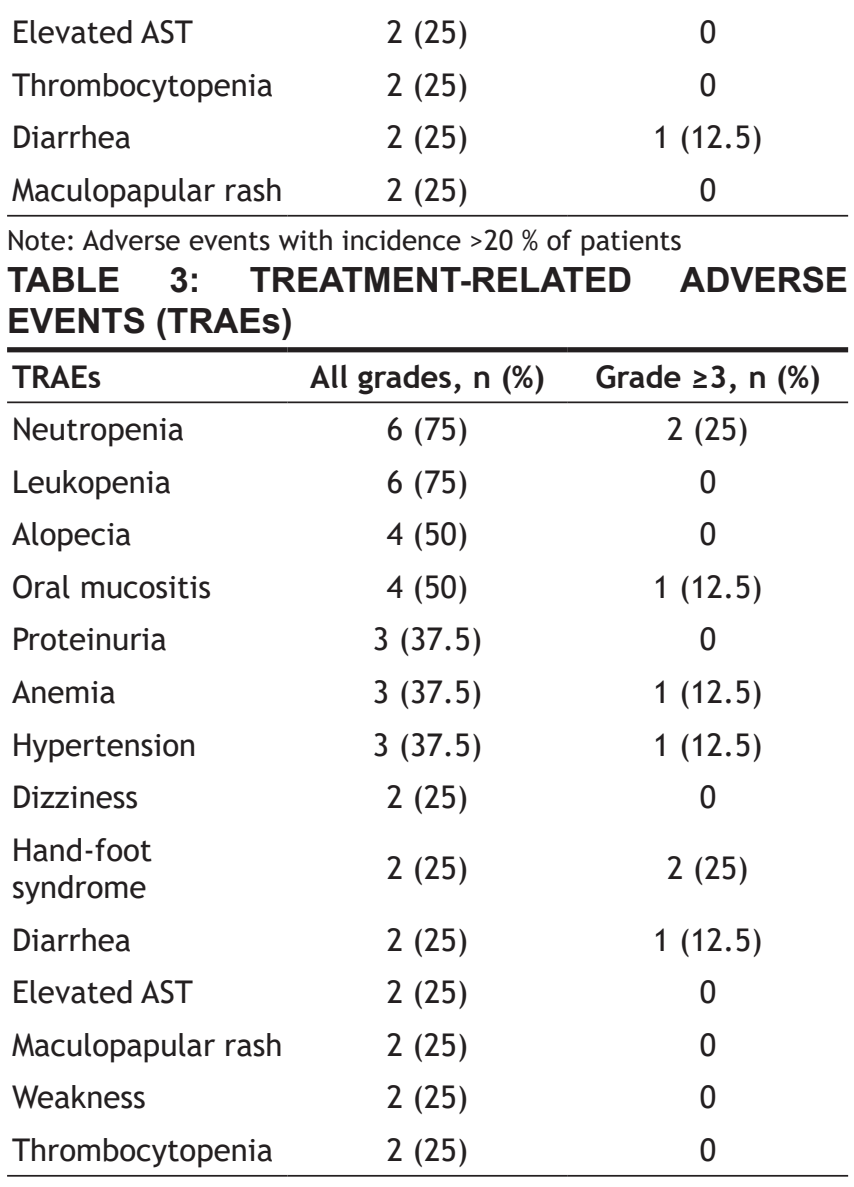

Note: TRAEs with incidence $>20 \%$ of patients

TABLE 4: EFFICACY

\section{Acknowledgement:}

The work was supported by National Natural Science Foundation of China (No.81803070) and Zhejiang Provincial Natural Science Foundation of China under Grant (No. LSY19H160006). The ClinicalTrials.gov Identifier Number: NCT03071042.

\section{Authors' contributions:}

Yu Zheng and Wei Wang had contributed equally to this work.

\section{Conflict of interests:}

The authors declared no conflicts of interest. 


\section{REFERENCES}

1. Bray F, Ferlay J, Soerjomataram I, Siegel RL, Torre LA, Jemal A. Global cancer statistics 2018: GLOBOCAN estimates of incidence and mortality worldwide for 36 cancers in 185 countries. CA Cancer J Clin 2018;68(6):394-424.

2. Feng RM, Zong YN, Cao SM, Xu RH. Current cancer situation in China: good or bad news from the 2018 Global Cancer Statistics? Cancer Communications 2019;39(1):1-2.

3. Fuchs CS, Tomasek J, Yong CJ, Dumitru F, Passalacqua R, Goswami C, et al. Ramucirumab monotherapy for previously treated advanced gastric or gastro-oesophageal junction adenocarcinoma (REGARD): an international, randomised, multicentre, placebo-controlled, phase 3 trial. Lancet 2014;383(9911):31-9.

4. Li J, Qin S, Xu J, Xiong J, Wu C, Bai Y, et al. Randomized, double-blind, placebo-controlled phase III trial of apatinib in patients with chemotherapy-refractory advanced or metastatic adenocarcinoma of the stomach or gastroesophageal junction. J Clin Oncol 2016;34(13):1448-54.

5. Kang YK, Boku N, Satoh T, Ryu MH, Chao Y, Kato K, et al. Nivolumab in patients with advanced gastric or gastrooesophageal junction cancer refractory to, or intolerant of, at least two previous chemotherapy regimens (ONO-4538-12, ATTRACTION-2): a randomised, double-blind, placebocontrolled, phase 3 trial. Lancet 2017;390(10111):2461-71.

6. Fuchs CS, Doi T, Jang RW, Muro K, Satoh T, Machado M, et al. Safety and efficacy of pembrolizumab monotherapy in patients with previously treated advanced gastric and gastroesophageal junction cancer: phase 2 clinical KEYNOTE-059 trial. JAMA Oncol 2018;4(5):e180013.

7. Ford HE, Marshall A, Bridgewater JA, Janowitz T, Coxon FY, Wadsley J, et al. Docetaxel versus active symptom control for refractory oesophagogastric adenocarcinoma (COUGAR-02): an open-label, phase 3 randomised controlled trial. Lancet Oncol 2014;15(1):78-86.

8. Hironaka S, Ueda S, Yasui H, Nishina T, Tsuda M, Tsumura $\mathrm{T}$, et al. Randomized, open-label, phase III study comparing irinotecan with paclitaxel in patients with advanced gastric cancer without severe peritoneal metastasis after failure of prior combination chemotherapy using fluoropyrimidine plus platinum: WJOG 4007 trial. J Clin Oncol 2013;31(35):443844.

9. Tian S, Quan H, Xie C, Guo H, Lü F, Xu Y, et al. YN968D1 is a novel and selective inhibitor of vascular endothelial growth factor receptor-2 tyrosine kinase with potent activity in vitro and in vivo. Cancer Sci 2011;102(7):1374-80.

10. Li J, Qin S, Xu J, Guo W, Xiong J, Bai Y, et al. Apatinib for chemotherapy-refractory advanced metastatic gastric cancer: results from a randomized, placebo-controlled, parallel-arm, phase II trial. J Clin Oncol 2013;31(26):3219-25.
11. Jiang Q, Zhang NL, Ma DY, Tan BX, Hu X, Fang XD. Efficacy and safety of apatinib plus docetaxel as the second or above line treatment in advanced nonsquamous NSCLC: A multicenter prospective study. Medicine 2019;98(26):e16065.

12. Guo Y, Tang J, Huang XE, Cao J. Efficacy and toxicity of apatinib combined with or without chemotherapy for patients with advanced or metastatic chemotherapy-refractory gastric adenocarcinoma: a prospective clinical study. Medicine 2019;98(6):e13908.

13. Zhang F, Yin Y, Ni T, Zhang M, Zhou Z, Sun X, et al. Treatment effect of apatinib combined chemotherapy as second-line or above therapy in patients with advanced gastric cancer or adenocarcinoma of the gastroesophageal junction. Pharmazie 2020;75(8):389-94.

14. Lu B, Lu C, Sun Z, Qu C, Chen J, Hua Z, Tong R, Zhang J. Combination of apatinib mesylate and second-line chemotherapy for treating gastroesophageal junction adenocarcinoma. J Int Med Res 2019;47(5):2207-14.

15. Zaanan A, Samalin E, Aparicio T, Bouche O, Laurent-Puig $\mathrm{P}$, Manfredi S, et al. Phase III randomized trial comparing 5-fluorouracil and oxaliplatin with or without docetaxel in first-line advanced gastric cancer chemotherapy (GASTFOX study). Dig Liver Dis 2018;50(4):408-10.

16. Albertsson M, Johansson B, Friesland S, Kadar L, Letocha H, Frykholm G, Wagenius G. Phase II studies on docetaxel alone every third week, or weekly in combination with gemcitabine in patients with primary locally advanced, metastatic or recurrent esophageal cancer. Med Oncol 2007;24(4):407-12.

17. Sym SJ, Hong J, Park J, Cho EK, Lee JH, Park YH, et al. A randomized phase II study of biweekly irinotecan monotherapy or a combination of irinotecan plus 5-fluorouracil/leucovorin (mFOLFIRI) in patients with metastatic gastric adenocarcinoma refractory to or progressive after first-line chemotherapy. Cancer Chemother Pharmacol 2013;71(2):481-8.

18. Wilke H, Muro K, Van Cutsem E, Oh SC, Bodoky G, Shimada $\mathrm{Y}$, et al. Ramucirumab plus paclitaxel versus placebo plus paclitaxel in patients with previously treated advanced gastric or gastro-oesophageal junction adenocarcinoma (RAINBOW): a double-blind, randomised phase 3 trial. Lancet Oncol 2014;15(11):1224-35.

This is an open access article distributed under the terms of the Creative Commons Attribution-NonCommercial-ShareAlike 3.0 License, which allows others to remix, tweak, and build upon the work non-commercially, as long as the author is credited and the new creations are licensed under the identical terms

This article was originally published in a special issue, "Therapeutic Perspectives in Biomedical Research and Pharmaceutical Sciences and their Nursing Methods"

Indian J Pharm Sci 2021:83(4)Spl issue "10-14" 